\title{
A Compact Quad-Ridge Orthogonal Mode Transducer with Wide Operational Bandwidth
}

\author{
A. W. Pollak, Michael E. Jones
}

\begin{abstract}
We present the design and the measured performance of a compact quad-ridge orthomode transducer (OMT) operating in $\mathrm{C}$-band with more than $100 \%$ fractional bandwidth. The OMT comprises two sets of identical orthogonal ridges mounted in a circular waveguide. The profile of these ridges was optimised to reduce significantly the transition length, while retaining the wide operational bandwidth of the quad-ridge OMT. In this letter, we show that the optimised compact OMT has better than $-15 \mathrm{~dB}$ return loss with the cross-polarisation well below $-40 \mathrm{~dB}$ in the designated $4.0-8.5 \mathrm{GHz}$ band.
\end{abstract}

Index Terms-Orthomode Transducer, Wideband performance, Quad-Ridged, Compact.

\section{INTRODUCTION}

A $\mathrm{N}$ orthomode transducer (OMT) is commonly used to extract the two orthogonally polarised modes from a waveguide, in the case of a circular waveguide, from the fundamental $\mathrm{TE}_{11}$ modes. Together with the feed horn, the OMT forms the transition from free-space waves to waves confined in coaxial cables.

The most commonly used high-performance OMTs employ planar probes [1], a turnstile junction [2], [3], Bøifot junction [4], [5], double ridged [6] or quad-ridged fins [7] to separate the polarised signal. The first two options are generally used for their simplicity, but for applications requiring more than 1.8:1 bandwidth, the quad-ridged design is preferable. Table I gives a brief overview of the fractional bandwidth and physical transition length of different OMT designs from the literature. All the presented OMTs have similar performance in cross-polarisation, return loss, and insertion loss within small variations. It can be seen that traditional OMTs are either compact and narrowband or physically long with a wideband performance.

For applications such as multi-band radio telescopes, the physical size of the OMT becomes an important part of the overall receiver design, as those receivers generally require cryogenic cooling. This becomes a challenging issue especially for the operation at $\mathrm{C}$-band $(4-8 \mathrm{GHz})$ and below, where traditional wideband OMTs form a large mass with respect to the overall receiver size. This can affect the design of an efficient cooling mechanism and can lead to an increased heat load.

The aim of this work is to design a compact quad-ridged OMT that has a transition length 5 times shorter than traditional designs, without compromising the broadband performance. The frequency range is set by the requirement to use

A. W. Pollak and M. E. Jones are with the Department of Physics (Astrophysics), University of Oxford, Denys Wilkinson Building, Keble Road, OX13RH, Oxford, UK. E-mail: Alexander.Pollak@Physics.ox.ac.uk

Manuscript received XXXX XX, 2015; revised XXXX XX, 2015.

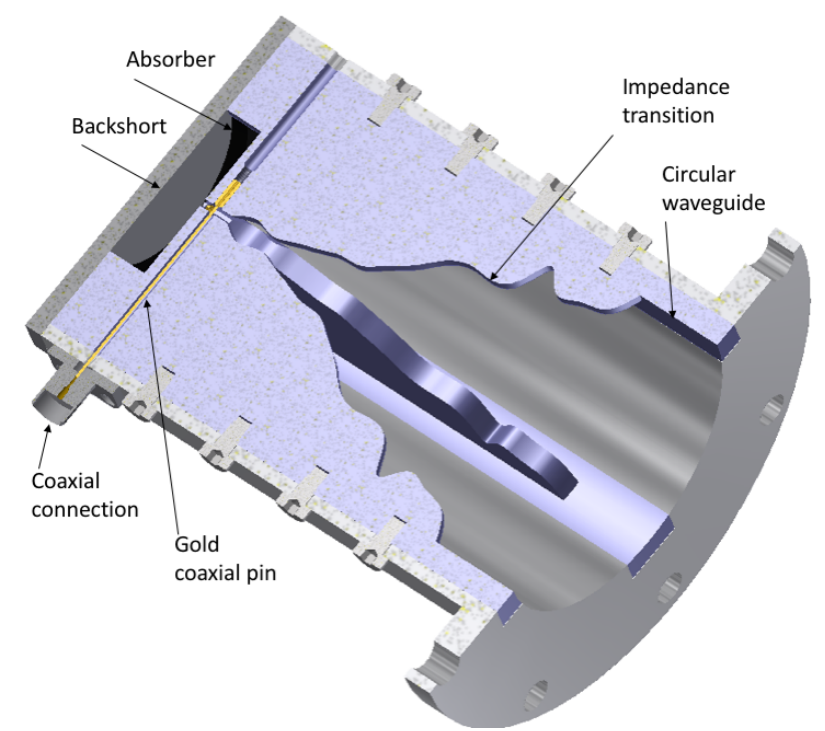

Fig. 1. Cross-sectional view of the OMT through the centre line. The four ridges are slid into the groove of the circular waveguide and held in place by radial screws. The golden coaxial pin is inserted through the hole opposite the SMA connector and set into position by the thread at the centre of the ridge.

TABLE I

COMPARISON OF FRACTIONAL BANDWIDTH AND TRANSITION LENGTHS FOR DIFFERENT OMT DESIGNS. $\lambda_{\mathrm{L}}$ : WAVELENGTH CORRESPONDING TO THE LOWEST OPERATIONAL FREQUENCY.

\begin{tabular}{lll}
\hline OMT type & Fractional bandwidth & Transition length \\
\hline This work & $2.1: 1$ & $0.8 \lambda_{\mathrm{L}}$ \\
Compact Broadband Planar [1] & $1.5: 1$ & $0.23 \lambda_{\mathrm{L}}$ \\
Turnstile-Based OMT[2] & $1.57: 1$ & $1.13 \lambda_{\mathrm{L}}$ \\
Turnstile-Based OMT[3] & $1.40: 1$ & $0.72 \lambda_{\mathrm{L}}$ \\
Bøifot-Based OMT[4] & $1.74: 1$ & $1.84 \lambda_{\mathrm{L}}$ \\
Double-Ridged OMT[6] & $1.63: 1$ & - \\
S-Band EVLA OMT [8] & $2.0: 1$ & $2.42 \lambda_{\mathrm{L}}$ \\
Quad-Ridged OMT [9] & $2.2: 1$ & $4.0 \lambda_{\mathrm{L}}$ \\
\hline
\end{tabular}

the receiver both for radio astronomy applications at $4-8 \mathrm{GHz}$, and for deep-space satellite communication at $8.4 \mathrm{GHz}$. The length reduction is achieved by altering and optimising the profile of the ridges to find the shortest design that would still retain better than $-15 \mathrm{~dB}$ return loss and $-40 \mathrm{~dB}$ crosspolarisation over the frequency range from $4-8.5 \mathrm{GHz}$.

\section{Design AND Simulation}

Since our OMT will be used with a C-band corrugated horn with a circular aperture and connected via two coaxial cables to the receiver, it needs to be optimised to serve two separate but interconnected purposes. Firstly, we need to 
TABLE II

PARAMETERS FOR THE TRANSITION BETWEEN THE QUAD-RIDGED STRUCTURE AND THE SMA CONNECTOR.

\begin{tabular}{lrl}
\hline Parameter & value & fixed/variable \\
\hline Waveguide radius & $24.4 \mathrm{~mm}$ & fixed \\
$\Delta_{1}$ & $0.254 \mathrm{~mm}$ & fixed \\
$\Delta_{2}$ & $0.59 \mathrm{~mm}$ & fixed \\
$\Delta_{3}$ & $3.6 \mathrm{~mm}$ & variable \\
$\Delta_{4}$ & $1.3 \mathrm{~mm}$ & variable \\
$\Delta_{5}$ & $0.48 \mathrm{~mm}$ & variable \\
\hline
\end{tabular}

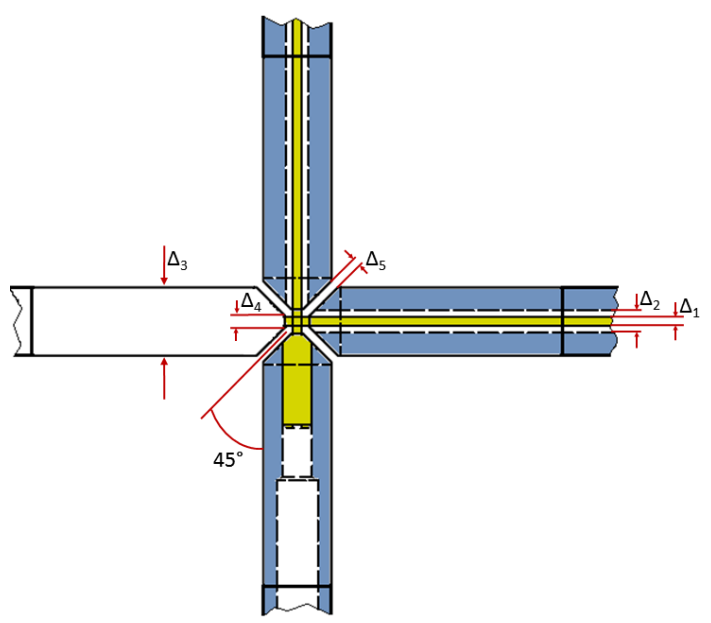

Fig. 2. Cross sectional view from bottom with the lid and backshort absorber removed. $\Delta_{1}$ and $\Delta_{2}$ are the fixed dimensions for the coaxial transmission line. $\Delta_{3}, \Delta_{4}$, and $\Delta_{5}$ are the free design parameters for the quad-ridged structure, the ridge thickness, the centre gap, and the gap at $45^{\circ}$ between the perpendicular ridges, respectively.

ensure that the energy from the $\mathrm{TE}_{11}$ modes propagating in the unloaded waveguide is transferred efficiently to the section with the quad-ridged fins. Secondly, we need to transfer the energy concentrated in the gap between the ridges to an SMA connector efficiently via a quarter-wavelength backshort.

We used a combination of MATLAB and Ansys's HFSS v.15 [10] (High Frequency Structural Simulator) for the design and optimisation process. MATLAB is used to define the HFSS 3D model, allowing it to be changed in a time saving manner, while HFSS is used to analyse and optimise the model. The flow diagram of this optimisation process is shown in Fig. 3, where blue and red represent the steps which were executed in Matlab and HFSS, respectively. The complete model is shown in Fig. 1 and the design of the transition between the quad-ridged structure and the SMA pin is shown in Fig 2. The dimensions of the coaxial probe (core diameter $\Delta_{1}$ and outer diameter $\Delta_{2}$ ) crossing the centre gap are fixed in order to match the $50 \Omega$ impedance of the SMA connector. Therefore, the free parameters to match the transition over the required bandwidth were the ridge thickness $\Delta_{3}$, the size of the centre gap $\Delta_{4}$, the gap at $45^{\circ}$ between the four perpendicular ridges $\Delta_{5}$, and the length and width of the backshort. The optimised dimensions for the presented OMT are shown in Table II.

Once the design and optimisation of the quad-ridged to SMA connector transition was complete, we proceeded to

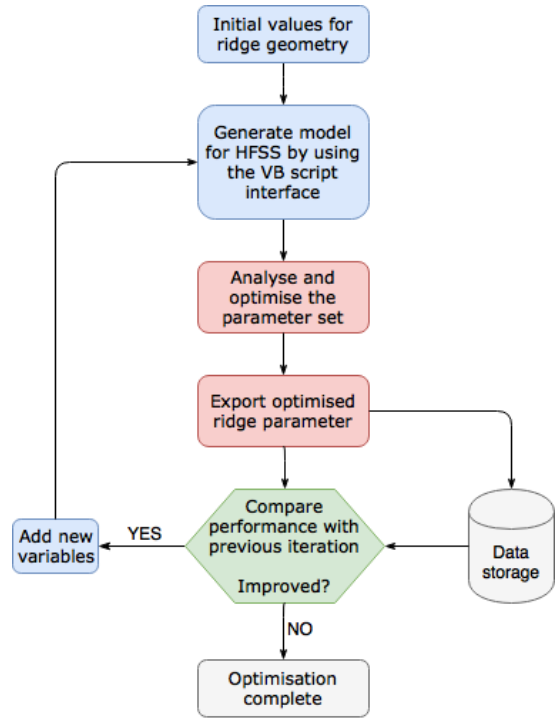

Fig. 3. The optimisation process used for the OMT. Shown in blue and red, the steps which were executed in Matlab and HFSS, respectively. Once the optimisation has been completed for a particular set of variables, we look at the result and decide whether to add more variables (green)

design and optimise the transition length between the field in the circular waveguide and the field concentrated in the centre gap. To achieve this, we used an iterative optimisation process based on a technique first presented in [11]. We begin by defining a set of parameters which describe the ridge geometry as a cubic spline interpolation between a number of points along the length of the ridge. The first and the last point in each set of parameters are fixed to match the waveguide radius and the final centre gap dimensions, respectively. Each of the remaining points can be chosen randomly within sensible constraints. To optimise a given set of parameters, we used the Sequential Non-linear Programming optimiser in HFSS v.15. This optimiser allows to specify a list of goals with individual weightings. We specified the simulated return loss, insertion loss $\left(\mathrm{TE}_{11}\right)$, cross-polarisation, and the amplitudes of three spurious modes $\left(\mathrm{TE}_{21}, \mathrm{TE}_{31}\right.$, and $\left.\mathrm{TM}_{11}\right)$ and applied a uniform weight to all goals.

Typically a few hundred iterations are required until the cost function converges to a minimum. This optimisation process starts initially with a small set of parameters, usually $2-4$, to find the general form of the ridge. We then retain this shape and add new variables, to form a larger set of parameters. All variables in the new parameter set are then optimised again to refine the ridge shape. This procedure is repeated until adding further variables does not lead to an improvement in performance.

Part of the design process was to investigate the mode excitation of the OMT, therefore we analysed trapped mode and propagating modes. Trapped mode or cavity mode resonances appear when there are higher-order modes present, which can only propagate within certain parts of the OMT and have no means to dissipate their energy. This is a known issue of quadridged OMTs and has been addressed before by adding an absorber at the backshort to dissipate their energy internally [8], [12]. Fig. 4 shows the simulated return loss and mode 


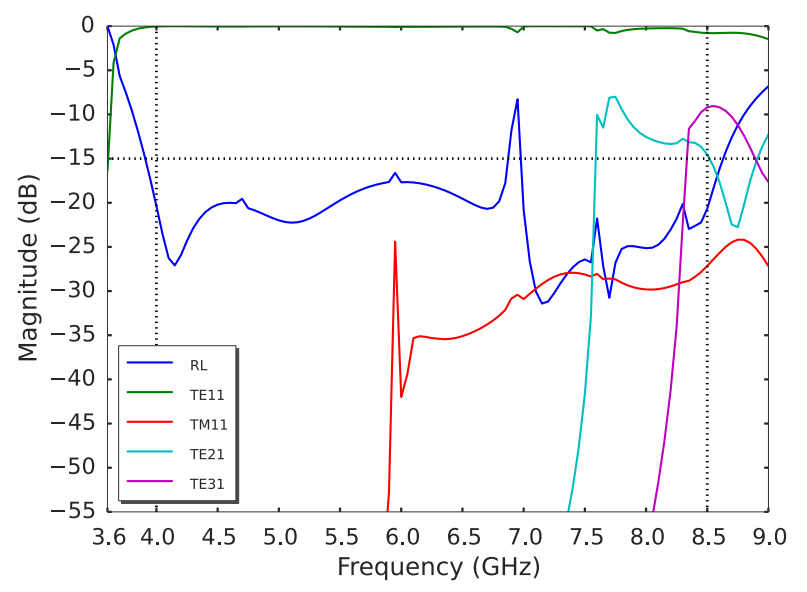

(a)

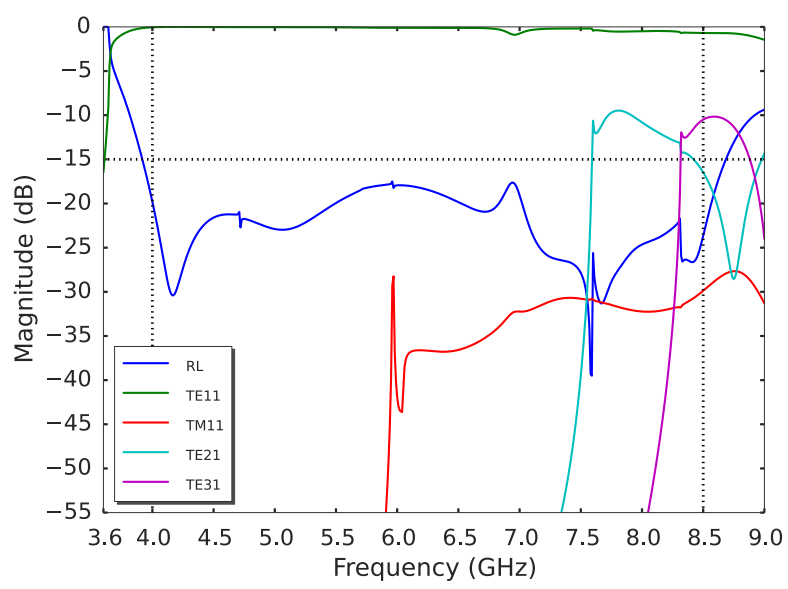

(b)

Fig. 4. Shows the simulated return loss and mode production of the OMT (a) without the absorber and (b) with the absorber present. The design band of the OMT is indicated by vertical lines and the requirement in return loss by the horizontal line.

production of the OMT with and without an absorber. Fig. 4a shows the appearance of trapped mode resonances which can be seen in the return loss curve at $4.7 \mathrm{GHz}, 5.9 \mathrm{GHz}$, and $6.9 \mathrm{GHz}$. In comparison to Fig. $4 \mathrm{~b}$, the resonances are diminished by the absorber and the return loss remains well below $-15 \mathrm{~dB}$. It is worth noting that the absorption of the resonance at $6.9 \mathrm{GHz}$ also increases the insertion loss at this frequency. Fig. 4 also shows the mode production of the OMT. It can be seen, that above $7.5 \mathrm{GHz}$ the magnitude of the $\mathrm{TE}_{21}$ and $\mathrm{TE}_{31}$ modes are above $-15 \mathrm{~dB}$ and as a result the insertion loss of the fundamental $\mathrm{TE}_{11}$ mode is increased.

\section{EXPERIMENTAL RESULTS}

To measure the performance of the compact OMT, we manufactured two identical OMTs from aluminium. The prototypes were manufactured in four steps using wire eroding technology, with a tolerance better than $50 \mu \mathrm{m}$. First, the waveguide including the grooves aligning the ridges was wire eroded. In the second step, the ridges without their final profile were fitted and aligned into the grooves and the holes for the coaxial probes were drilled. In the third step, the centre

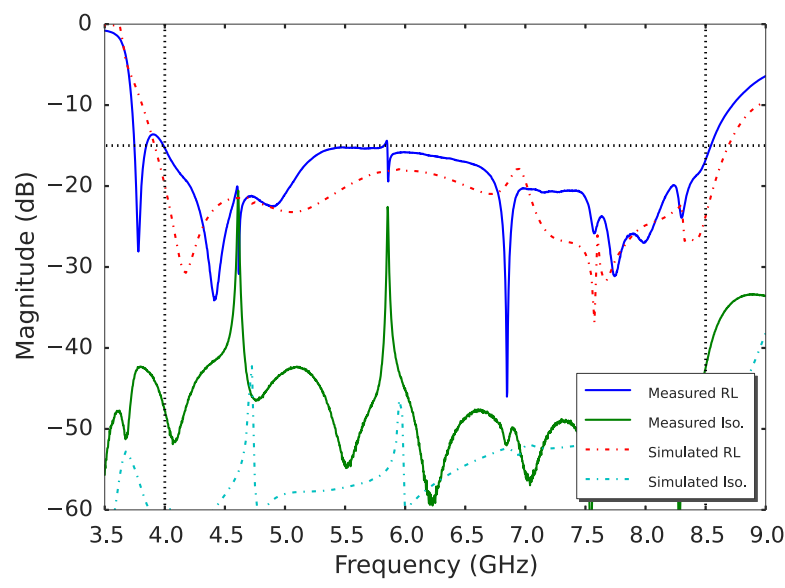

Fig. 5. Simulated and measured characteristics of an OMT, terminated with a waveguide load. Traces plotted are return loss and isolation between the two coaxial ports. The solid lines show the measured responses and the dasheddotted-dashed lines show the simulated performances. The design band of the OMT is indicated by vertical lines and the requirement in return loss by the horizontal line.

gap, the final ridge thickness and chamfer were wire eroded. Finally, the ridges were removed and the varying height profile was wire cut along the ridge length.

All measurements were performed using an Anritsu 37369C Vector Network Analyser (VNA) which was calibrated to its coaxial ports. The return loss and isolation were measured by connecting the two VNA ports to each polarisation port of the OMT, with the waveguide port terminated by a circular waveguide load with an integrated absorber spear in its centre. We treated this termination as an ideal absorber, therefore no calibration was applied to remove any reflections from this termination method. In Fig. 5, we show the simulated and measured return loss and isolation performance of the OMT. It can be seen that the measured return loss is below $-15 \mathrm{~dB}$ over the entire frequency range from $4-8.5 \mathrm{GHz}$. The isolation remains well below $-40 \mathrm{~dB}$ except for two spikes at $4.7 \mathrm{GHz}$ and $5.9 \mathrm{GHz}$ which correspond to trapped-mode resonances in the OMT structure that were not completely eliminated by the backshort absorber.

The insertion loss and the cross-polarization coefficient were measured by connecting both OMTs at their circular waveguide ports. As we can not measure the insertion loss of a single OMT directly, we modelled the measurement setup using both OMTs and compared the predicted results with our measurement. This allows us to consider the setup as a 4-port device, where the combined insertion loss is represented by the $\mathrm{S}$ parameters $\mathrm{S}_{31}, \mathrm{~S}_{42}$ and the combined cross-polarisation by $\mathrm{S}_{41}, \mathrm{~S}_{32}$. For each $\mathrm{S}$ parameter measurement, the unused ports were terminated using two $50 \Omega$ SMA terminations. In Fig. 6, we show the insertion loss measured for both polarisations in comparison to the predicted response of the model. It can be seen that the measured performance of the OMT setup agrees very well with the simulation. The turquoise dashed-dotteddashed line shows the predicted insertion loss for a single OMT. The dip seen at $6.9 \mathrm{GHz}$ corresponds to the absorbed trapped mode resonance at this frequency and the increase in 


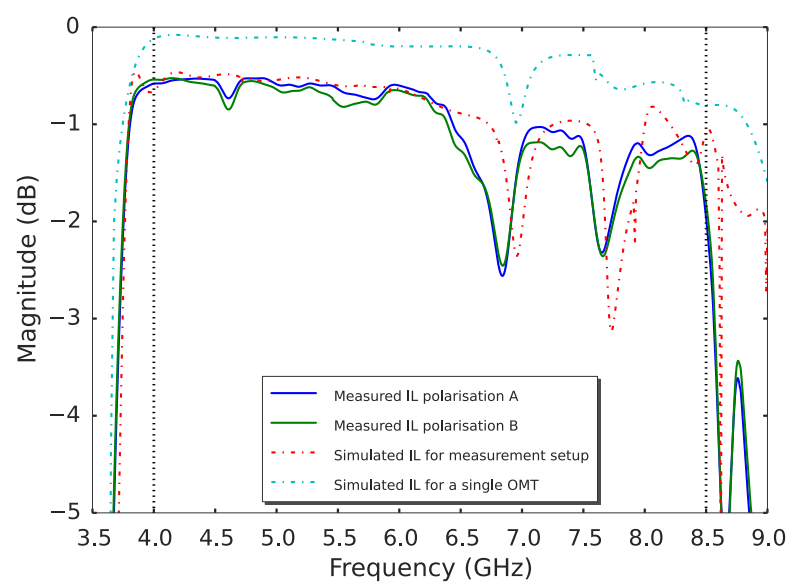

Fig. 6. Simulated and measured insertion loss of the measurement setup. Traces plotted are the measured insertion loss for both polarization $\mathrm{A}$ and $\mathrm{B}$ from the coaxial port to the fundamental $\mathrm{TE}_{11}$ mode, the simulated insertion loss of the measurement setup and the simulated performance of a single OMT. The design band of the OMT is indicated by vertical lines.

insertion loss above $7.5 \mathrm{GHz}$ is due to the occurrence of the $\mathrm{TE}_{21}$ and $\mathrm{TE}_{31}$ mode.

To measure the cross-polarisation, we used the same test setup and simply interchanged the $50 \Omega$ SMA termination with the VNA port on one of the two OMTs. The measured transmission between the two VNA ports now correspond to the combined cross-polarisation of the measurement setup. Fig. 7 shows the measured cross-polarisation in comparison to the predicted response, plotted in the same way as for the insertion loss previously. One notes immediately the existence of a number of sharp resonances in both the measurement and the simulation, which are caused by reflections within the cavity of the measurement setup.

However, the number and amplitude of these resonances is reduced dramatically if only a single OMT is simulated (see the red dashed-dotted line in Fig. 7). The remaining two spikes at $4.7 \mathrm{GHz}$ and $5.9 \mathrm{GHz}$, correspond to the same trapped mode resonances as described earlier. Since in practical applications, only one OMT is needed, we are confident that these resonances would not affect its performance. It is also worthwhile to note that the cross-polarisation performance it well below $-40 \mathrm{~dB}$, which is more than sufficient for general purpose C-band OMTs.

\section{CONCLUSION}

A novel design of a compact quad-ridged OMT with optimised ridge profile has been presented. We reduced the transition length by a factor of 5 compared to traditional wideband OMTs while retaining a fractional bandwidth of 2.1:1. Two prototypes have been manufactured and tested in the frequency range $4-8.5 \mathrm{GHz}$, and we found excellent agreement between the measured and simulated performance. The measured crosspolarisation is low at $-40 \mathrm{~dB}$ and indicates that the simulated cross-polarisation of a single OMT of less than $-50 \mathrm{~dB}$ is possible. The measured return loss remains below $-15 \mathrm{~dB}$ over the whole frequency band.

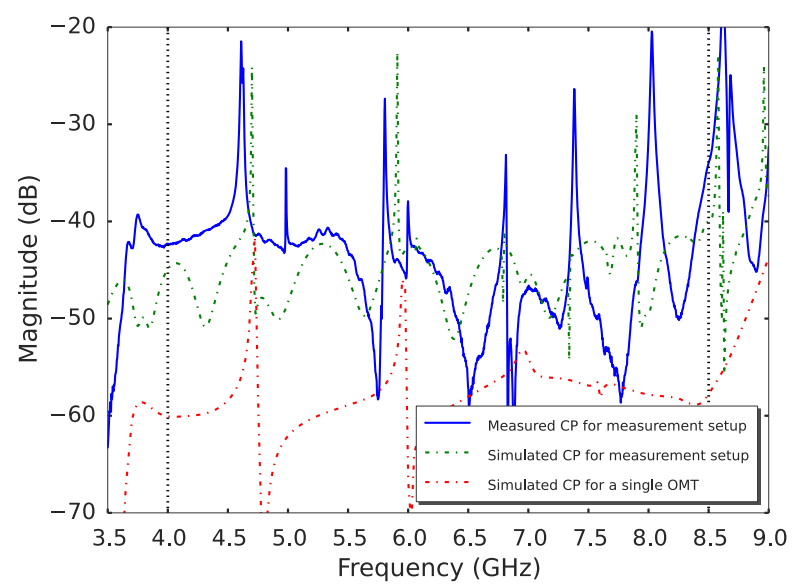

Fig. 7. Simulated and measured cross-polarisation of the measurment setup. Traces plotted are measured and the simulated cross-polarisation of the measurement setup, and the simulated performance of a single OMT. The design band of the OMT is indicated by vertical lines.

\section{ACKNOWLEDGMENT}

The OMT presented in this paper was developed for the Consortium of Universities for Goonhilly Astronomy (CUGA)[13] and for the Goonhilly Earth Station (GES), supported by an STFC CASE studentship.

\section{REFERENCES}

[1] P. K. Grimes, O. G. King, G. Yassin, and M. E. Jones, "Compact broadband planar orthomode transducer," Electronics Letters, vol. 43, no. 21, pp. 1146-1147, Oct 2007.

[2] A. Tribak, J. L. Cano, A. Mediavilla, and M. Boussouis, "Octave bandwidth compact turnstile-based orthomode transducer," IEEE Microwave and Wireless Components Letters, vol. 20, no. 10, pp. 539-541, Oct 2010.

[3] Y. Aramaki, N. Yoneda, M. Miyazaki, and T. Horie, "Ultra-thin broadband omt with turnstile junction," in IEEE MTT-S International Microwave Symposium Digest, 2003, vol. 1, June 2003, pp. 47-50 vol.1.

[4] J. A. Ruiz-Cruz, J. R. Montejo-Garai, J. M. Rebollar, and J. M. Montero, "C-band orthomode transducer for compact and broadband antenna feeders," Electronics Letters, vol. 45, no. 16, pp. 813-814, July 2009.

[5] R. Banham, G. Valsecchi, L. Lucci, G. Pelosi, S. Selleri, V. Natale, R. Nesti, and G. Tofani, "Electroformed front-end at $100 \mathrm{ghz}, " 2005$.

[6] A. Dunning, "Double ridged orthogonal mode transducer for the 16$26 \mathrm{ghz}$ microwave band," in Workshop on the Applications of Radio Science, Feb 20-22, 2002.

[7] D. I. L. D. Villiers, P. Meyer, and K. D. Palmer, "Broadband offset quadridged waveguide orthomode transducer," Electronics Letters, vol. 45, no. 1 , pp. 60-62, January 2009.

[8] G. M. Coutts, H. Dinwiddie, and P. Lilie, "S-band octave-bandwidth orthomode transducer for the expanded very large array," in 2009 IEEE Antennas and Propagation Society International Symposium, June 2009, pp. 1-4.

[9] Z. Zhuang, B. Li, and Q. Fan, "Design of improved quad-ridged orthomode transducer," in 2010 International Conference on Microwave and Millimeter Wave Technology, May 2010, pp. 867-870.

[10] ANSYS, Inc., "Hfss." [Online]. Available: http://www.ansys.com/Products/Electronics/ANSYS-HFSS

[11] C. A. W. Vale and P. Meyer, "Designing high-performance finline tapers with vector-based optimization," IEEE Transactions on Microwave Theory and Techniques, vol. 47, no. 12, pp. 2467-2472, Dec 1999.

[12] G. M. Coutts, "Wideband diagonal quadruple-ridge orthomode transducer for circular polarization detection," IEEE Transactions on Antennas and Propagation, vol. 59, no. 6, pp. 1902-1909, June 2011.

[13] C. of Universities for Goonhilly Astronomy. (2017) Goonhilly - repurposing of communications antennas for radio astronomy. [Online]. Available: https://www2.physics.ox.ac.uk/research/experimental-radiocosmology/goonhilly 